\title{
Carcinome épidermoïde développé autour d'un implant : un cas de retard diagnostique lié à une absence d'analyse histologique puis à un faux négatif
}

\author{
Marie-Alix Fauroux, Charlotte Koren, Jacques-Henri Torres \\ Faculté d'Odontologie, 545 avenue Pr JL Viala, 34193 Montpellier cedex 5, France \\ mariealix.fauroux@free.fr
}

Les carcinomes des voies aérodigestives supérieures se situent au $4^{\mathrm{e}}$ rang des cancers les plus fréquents pour le sexe masculin; les principaux facteurs de risque sont l'alcool, le tabac et les infections à papillomavirus. Par ailleurs, les implants dentaires représentent une thérapeutique de plus en plus populaire. Peu de cas de carcinomes épidermoïdes développés autour d'implants dentaires ont été rapportés (Gallego et al. 2008, Meijer et al. 2010). Les implants pourraient constituer un facteur irritant et/ou un cofacteur inflammatoire (Jane-Salas et al. 2011). Mais le rôle cancérigène n'a pas été clairement établi (Abu El-Naaj et al. 2007, Bhatavadekar 2011) et une simple coïncidence topographique ne peut être écartée

Un patient âgé de 73 ans, en bonne santé générale, non fumeur, édenté total, porteur d'une prothèse mandibulaire stabilisée par une barre supportée par 3 implants symphysaires, a consulté son chirurgien-dentiste traitant pour une lésion gingivale bourgeonnante de $2 \mathrm{~cm}$ de diamètre, apparue autour d'un implant. Un cliché rétro-alvéolaire montrait une cratérisation osseuse autour de l'implant situé du côté droit. Le praticien a déposé l'implant concerné et excisé la lésion, sans demander d'analyse histologique.

La lésion ayant récidivé en 15 jours, le praticien a adressé le patient dans le service. L'examen objectivait une tuméfaction gingivale sessile, mesurant plus de $2 \mathrm{~cm}$, inhomogène, bourgeonnante et kératosique. Une première biopsie emportant à la fois une partie de la lésion et une zone cliniquement saine a conclu à une hyperplasie épithéliale verruqueuse sans signe de dysplasie. Une exérèse complète a été réalisée dans un deuxième temps, avec une marge de sécurité. L'examen histologique de la pièce d'exérèse a montré qu'il s'agissait d'un carcinome épidermoïde différencié kératinisant. Le patient a alors été orienté vers le service d'ORL. Après un bilan d'extension, il a subi une né pelvi-glossectomie antéro-latérale droite avec un curage ganglionnaire homolatéral, sans radiothérapie complémentaire. Après un an de silence clinique, le patient reste l'objet d'une surveillance régulière.

Le cas de ce patient rappelle l'absolue nécessité de l'analyse histologique de toute pièce d'exérèse, quel que soit son aspect clinique. En l'occurrence, on peut imaginer qu'un examen anatomopathologique de la lésion initiale aurait conduit à un diagnostic de malignité plus rapide. Par ailleurs, lorsque le résultat histologique ne coïncide pas avec l'aspect clinique de la lésion, il ne faut pas hésiter à pratiquer une biopsie (ou une exérèse) complémentaire plus profonde, multiple ou de localisation différente. Dans le cas rapporté, le diagnostic d'hyperplasie sans signe de dysplasie (faux négatif) ne cadrait pas avec la sémiologie clinique qui évoquait fortement un carcinome épidermoïde.

Il faut enfin souligner le rôle crucial de l'odontologiste dans le dépistage précoce des cancers buccaux. Le diagnostic d'une lésion maligne doit être évoqué devant toute prolifération tissulaire, y compris au voisinage d'un implant, où elle doit être rapidement différenciée d'une péri-implantite. 